\title{
Exploring the biocombinatorial potential of benzoxazoles: generation of novel caboxamycin derivatives
}

\author{
Armando A. Losada, Carmen Méndez, José A. Salas and Carlos Olano* (1)
}

\begin{abstract}
Background: The biosynthesis pathway of benzoxazole compounds caboxamycin and nataxazole have been recently elucidated. Both compounds share one of their precursors, 3-hydroxyanthranilate (two units in the case of nataxazole). In addition, caboxamycin structure includes a salicylate moiety while 6-methylsalycilate is the third scaffold in nataxazole. Pathways cross-talk has been identified in caboxamycin producer Streptomyces sp. NTK937, between caboxamycin and enterobactin pathways, and nataxazole producer Streptomyces sp. Tü6176, between nataxazole and coelibactin pathways. These events represent a natural form of combinatorial biosynthesis.

Results: Eleven novel caboxamycin derivatives, and five putative novel derivatives, bearing distinct substitutions in the aryl ring have been generated. These compounds were produced by heterologous expression of several caboxamycin biosynthesis genes in Streptomyces albus $\mathrm{J} 1074$ (two compounds), by combinatorial biosynthesis in Streptomyces sp. NTK937 expressing nataxazole iterative polyketide synthase (two compounds) and by mutasynthesis using a nonproducing mutant of Streptomyces sp. NTK937 (12 compounds). Some of the compounds showed improved bioactive properties in comparison with caboxamycin.

Conclusions: In addition to the benzoxazoles naturally biosynthesized by the caboxamycin and nataxazole producers, a greater structural diversity can be generated by mutasynthesis and heterologous expression of benzoxazole biosynthesis genes, not only in the respective producer strains but also in non-benzoxazole producers such as S. albus strains. These results show that the production of a wide variety of benzoxazoles could be potentially achieved by the sole expression of $c b x B C D E$ genes (or orthologs thereof), supplying an external source of salicylate-like compounds, or with the concomitant expression of other genes capable of synthesizing salicylates, such as cbxA or natPK.
\end{abstract}

Keywords: Gene expression, Heterologous expression, Nataxazole, Polyketide, Streptomyces

\section{Background}

Sequencing of Streptomyces sp. NTK937 genome, a deepsea sediment strain, revealed up to 35 putative gene clusters for the biosynthesis of secondary metabolites [1], including that for the biosynthesis of antibiotic caboxamycin. This compound belongs to the family of benzoxazoles [2], which includes compounds such as calcimycin [3], nataxazole [4], and A33853 [5], whose respective

\footnotetext{
*Correspondence: olanocarlos@uniovi.es

Departamento de Biología Funcional e Instituto Universitario de Oncología del Principado de Asturias (I.U.O.P.A), Universidad de Oviedo, C/Julian Claveria s/n, 33006 Oviedo, Asturias, Spain
}

biosynthesis pathways have been characterized [6-9]. The benzoxazole scaffold is known to bear different pharmacological properties such as being cytotoxic and/or antibiotic [10], possessing anti-leishmanial properties [11], or inhibiting the replication of hepatitis $C$ virus [12].

Recently, our group has elucidated the pathway for caboxamycin biosynthesis comprising nine structural genes, $c b x A-I$ (Fig. 1a). Caboxamycin derives from chorismate through the generation, activation and condensation of two precursors, 3-hydroxyanthranilate (3HAA) and salicylate (SA) [13]. Five of the estructural genes, $c b x A, c b x B, c b x D$ and $c b x E$, encoding a salicylate synthase, 3-oxoacyl-ACP-synthase, ACP, and amidohydrolase 


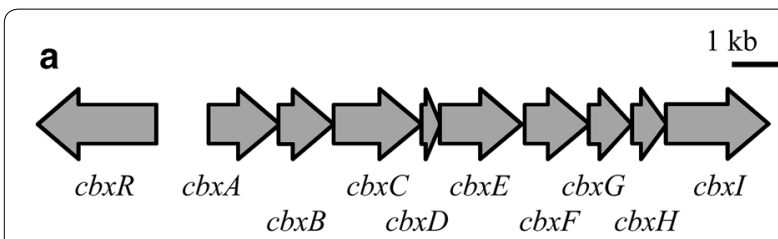

b

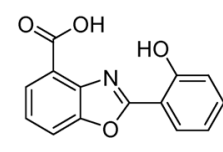

1

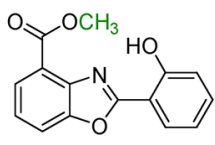

2

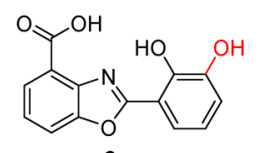

3
Fig. 1 Caboxamycin biosynthesis gene cluster and chemical structure of related compounds. a Genetic organization of caboxamycin biosynthesis gene cluster. $\mathbf{b}$ Chemical structure of caboxamycin, $\mathbf{1}$; O-methylcaboxamycin, 2; and 3'-hydroxycaboxamycin, $\mathbf{3}$

respectively, were found essential for caboxamycin biosynthesis. The remaining structural genes, $c b x C, c b x F, c b x G$, $c b x H$ and $c b x I$, encoding a AMP-dependent synthetaseligase, 3-deoxy-D-arabinohept-2-ulosonate-7-phosphate synthase, 2,3-dihydro-2,3-dihydroxybenzoate dehydrogenase, isochorismatase and anthranilate synthase respectively, have paralogs distributed throughout Streptomyces sp. NTK937 genome and thus they are not essential for the biosynthesis of caboxamycin. In addition, the biosynthesis gene cluster contains a positive SARP-like transcriptional regulator, $c b x R$. In this previous study, Streptomyces sp. NTK937 has been shown to naturally produce two different compounds: caboxamycin (1) and its methyl ester, $O$-methylcaboxamycin (2) (Fig. 1b), whose biosynthesis depends on an uncharacterized $O$-methyltransferase located neither within nor near the cluster. Furthermore, upon gene replacement of the salicylate synthase $c b x A$, a third compound was observed, $3^{\prime}$-hydroxycaboxamycin (3) (Fig. 1b), stemming from the cross-talk between the caboxamycin biosynthesis pathway and 2,3-dihydroxybenzoate (DHB) generated in the biosynthetic pathway for the siderophore enterobactin [13]. This phenomenon, together with our previous studies of the nataxazole biosynthetic cluster, where we identified cross-talk between the nataxazole and coelibactin biosynthesis pathways that led to biosynthesis of benzoxazole UK-1 [7, 8], sparked our interest for the generation of novel benzoxazoles exploring the biocombinatorial potential of these family of compounds.

In this work we report the generation of 16 novel caboxamycin derivatives (4-19) bearing distinct substitutions in the aryl ring have been generated. These compounds were produced by using different molecular approaches such as heterologous expression of selected genes, combinatorial biosynthesis using genes from different pathways and feeding different precursors to selected mutants by mutasynthesis.

\section{Results}

Heterologous expression of caboxamycin biosynthesis genes in Streptomyces albus $\mathrm{J} 1074$

The structural genes from the caboxamycin biosynthesis cluster, $c b x A B C D E F G H I$, have previously been expressed as a whole in Streptomyces lividans JT46 as heterologous host, leading to the production of caboxamycin [13]. Since paralogs to cbxFGHI exist in Streptomyces sp. NTK937 (probably involved in primary metabolism) we assumed that a similar situation could occur in other Streptomyces spp. Subsequently we aimed to induce the heterologous production of caboxamycin by solely expressing the $c b x A B C D E$ genes, encoding a salicylate synthase, oxoacyl-ACP synthase, AMP-dependant synthetase-ligase, ACP and amidohydrolase respectively, in two Streptomyces albus strains: S. albus J1074 [14] and B29 [15]. The former produces the glycosylated antibiotic family of paulomycins that involves the participation of genes plm15-18, orthologs to $c b x F G H I$ and responsible for the biosynthesis of the 3HAA-derived core of paulomycins [16]. On the other hand, in S. albus B29, genes plm15-16, orthologs of $c b x H I$, have been replaced by an apramycin resistance cassette, thus blocking paulomycin biosynthesis [15]. Expression of $c b x A B C D E$ under the control of constitutive promoter $\operatorname{erm} E^{*} p$ [17] using a pSET152-derived plasmid, pSET-cbxABCDE, afforded a substantial production of $\mathbf{1}$ in both strains (Fig. 2), clearly showing the existence of orthologs for $c b x F G H I$ within primary metabolism genes in the host strains. In addition, two novel products were generated (Fig. 2): compound $\mathbf{4}$, a benzoxazole-hydroxylated version of $\mathbf{1}$, barely detectable in the J1074 strain, but clearly produced in the B29 strain (Additional file 2: Table S1, Additional file 3: Figure S3); and 5, a $p$-aminobenzoate (PABA) amidelinked to a salicylate moiety, present in both strains (Additional file 2: Table S2, Additional file 3: Figure S4).

\section{Heterologous expression of natPK in Streptomyces sp. NTK937}

Within the scope of our research, ortholog genes from the related biosynthetic cluster of benzoxazole nataxazole [7] were heterologously expressed in several Streptomyces sp. NTK937 caboxamycin non-producing mutants, successfully restoring caboxamycin biosynthesis in all cases, except for the amidohydrolase coding natAM [13]. However, the nataxazole cluster also contains a structural gene with no ortholog in caboxamycin biosynthesis, $n a t P K$, an iterative type I polyketide synthase responsible for the biosynthesis of the 6-methylsalicylate (6MSA) moiety present in nataxazole. This gene was expressed, using plasmid pnatPK, in the Streptomyces sp. NTK937 non-producing strain $\triangle \mathrm{cbxA}$, thus generating noticeable quantities of the precursor 6MSA (Fig. 3). Furthermore, 


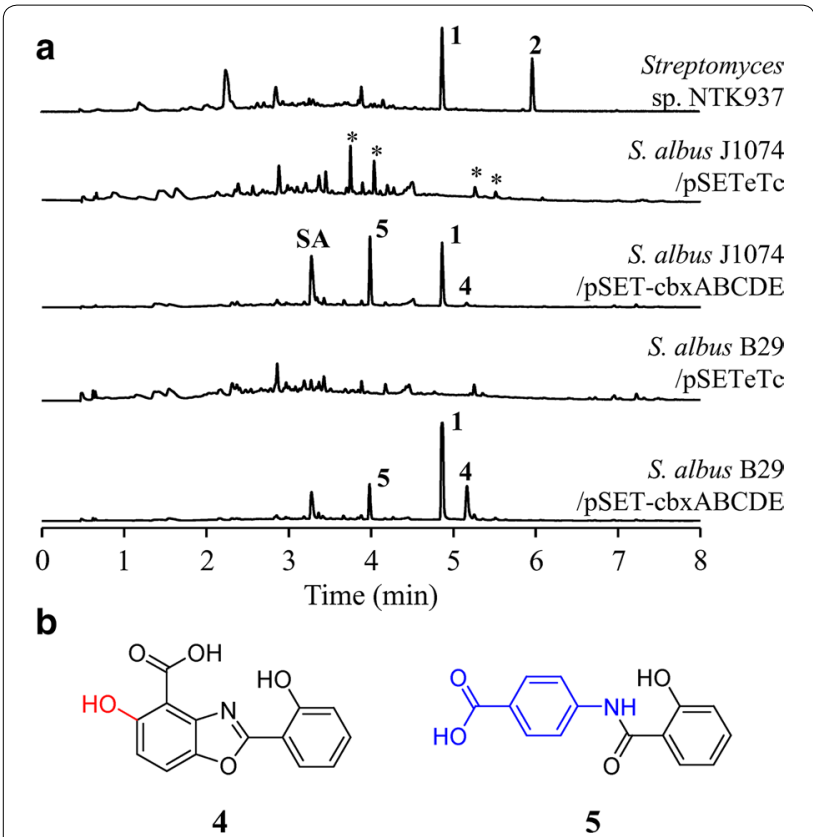

Fig. 2 Compounds generated by heterologous expression of caboxamycin biosynthesis genes in S. albus J1074. a UPLC analysis of Streptomyces sp. NTK937 wild-type and S. albus strains J1074 and B29 harboring pSET-cbXABCDE or control plasmid PSETeTc. Chromatograms were analyzed at $330 \mathrm{~nm}$. SA stands for salicylic acid. Asterisks indicate paulomycins and paulomenols. $\mathbf{b}$ Chemical structure of novel compounds $\mathbf{4}$ and $\mathbf{5}$

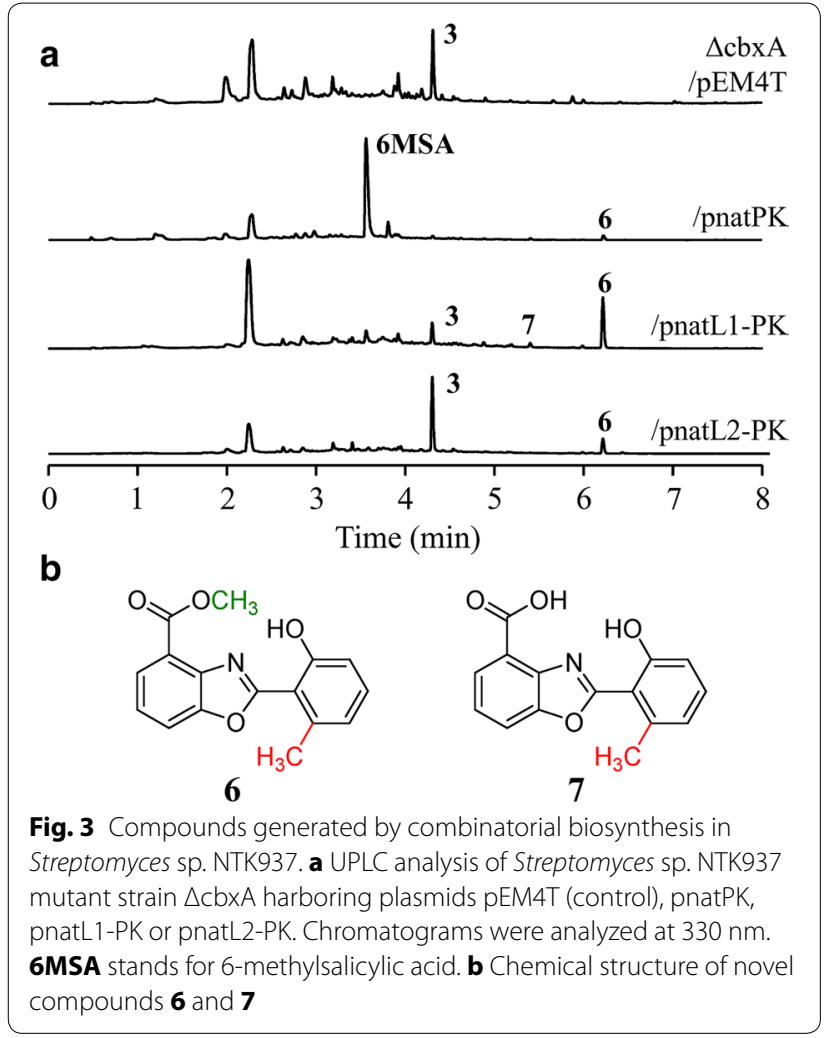

low levels of a caboxamycin derivative $(\mathbf{6})$ were produced as a consequence of combinatorial biosynthesis. Compound 6 carries both the aryl methylation characteristic of 6MSA, plus the methyl esterification ascribed to the unlocated $O$-methyltransferase (Additional file 2: Table S3, Additional file 3: Figure S5). We sought to improve production of 6 using plasmid constructs bearing both natPK and one of the AMP-dependant synthetase-ligases from the nataxazole cluster. The combined expression of natL1-natPK (pnatL1-PK) afforded the production of highest amounts of $\mathbf{6}$, whereas simultaneous expression of natL2-natPK (pnatL2-PK) favored instead the production of 3.

A small peak observed in $\Delta \mathrm{cbxA} /$ pnatL1-PK cultures, compound 7, with a retention time of $5.4 \mathrm{~min}$ (Fig. 3), possibly corresponds to the acid version of $\mathbf{6}$, given its coherently shorter than 6 (6.15 $\mathrm{min})$ but higher than $\mathbf{1}$ retention time $(4.90 \mathrm{~min})$. This peak was consistently observed along the different experiments performed, but its drastically low levels of production prevented its isolation and spectroscopic characterization, although HPLC-MS analysis is coherent with this hypothesis, showing a mass of $m / z 270.07[M+\mathrm{H}]^{+}$.

\section{Generation of novel derivatives by mutasynthesis}

Upon observing how amenable Streptomyces sp. NTK937 was to the combination of similar precursors to yield novel caboxamycins, we sought to obtain novel compounds following a mutasynthesis approach, a strategy involving the addition of modified precursors in biosynthetically-blocked mutants in order to afford novel derivatives of the original product. As a host, the Streptomyces sp. NTK937 non-producing double mutant strain $\triangle \mathrm{cbxA} /$ $\Delta \mathrm{entC}$ was selected, since syntheses of both SA and DHB are abolished, thus allowing for better resource allocation towards the production of novel caboxamycins.

A selection of different precursors, all bearing carboxylate groups attached to cyclic molecules, was initially tested and it was found that under standard working conditions, the mutant strain only assimilates molecules of salicylate structure, i.e., aromatic acids with hydroxyl substitution in position 2, but allowing for extra substitutions in other places. We selected an array of salicylates bearing an additional individual substitution with a hydroxyl, methoxyl, methyl or chlorine group in all available positions, plus salicylates with an heteroatomic substitution with nitrogen in any position within the ring (pyridinic acids) (Additional file 1: Figure S1), and added them at $1 \mathrm{mM}$ final concentration to the respective cultures, resulting in the production of several novel compounds, 8-19 (Figs. 4, 5) (Additional file 2: Figure S2, Tables S4-S11; Additional file 3: Figures S6-S13). 


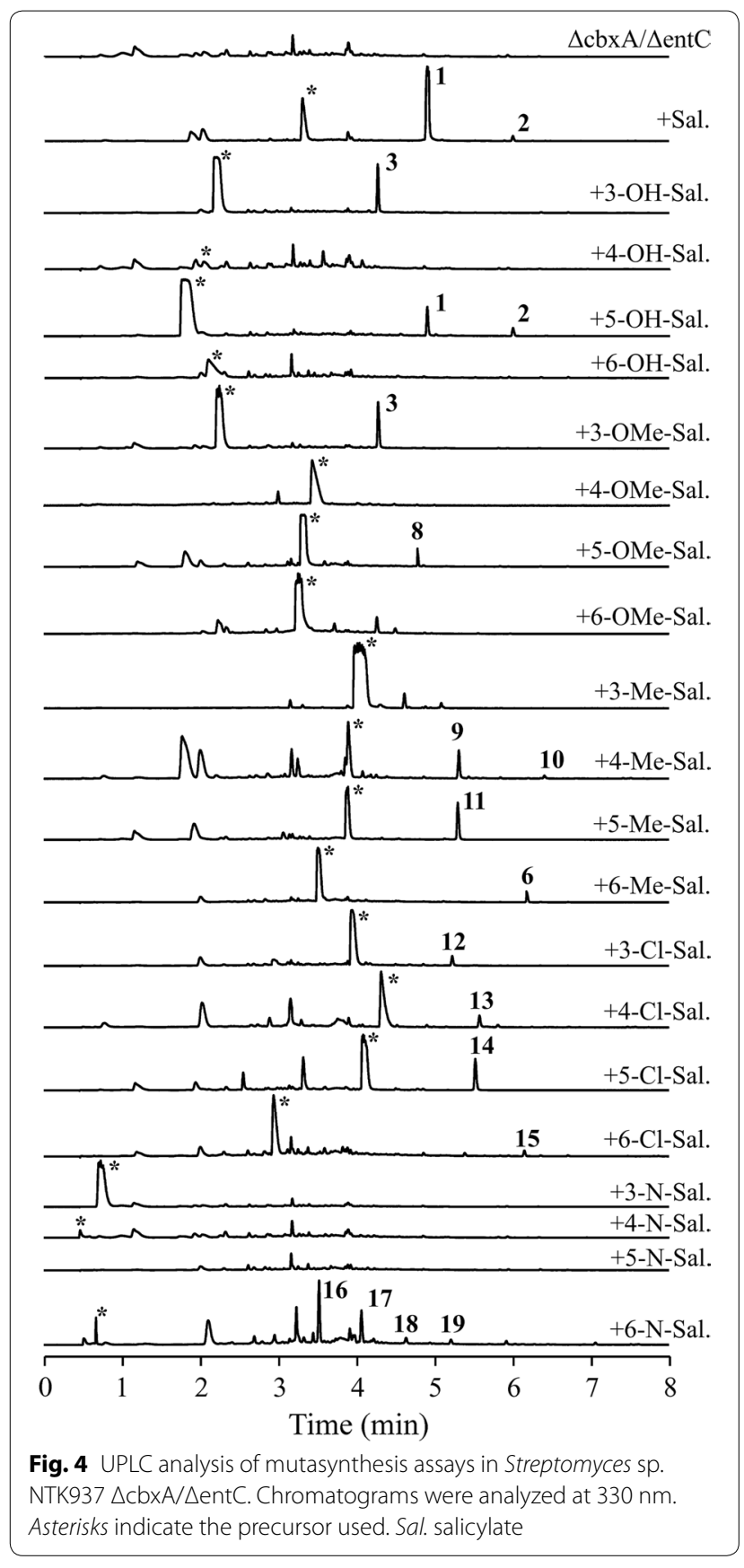

As expected, some of these compounds led to already characterized caboxamycins, such as the addition of SA behaving as a chemical complementation, restoring production of $\mathbf{1}$ and 2; DHB yielding 3; or 6MSA resulting in 6 (but, again, barely a hint of the putative acid form $7)$. On the other hand, some reagents spawned no benzoxazoles in our culture conditions, such as 4-methoxysalicylate, which also seems to exert some hindering effect on the growth of this strain. Similarly, none of the pyridinic acids resulted in novel benzoxazoles, with the exception of 3-hydroxypicolinate (3HPA), a moiety shared by related benzoxazole A33853 [9], with the remarkable difference that here it appears as compounds 16 and 18, sporting a complete benzoxazole feature, as opposed to the open, amide-linked structure of A33853. Furthermore, compounds 17 and 19, which, like 5, are PABA-derived, were unexpectedly obtained by the incorporation of 3HPA, a feat not readily observed with any of the other salicylates used in these assays. Moreover, it is unknown whether the methylation on the carboxylate in 19 could be a consequence of the same esterifying enzyme responsible for the biosynthesis of $\mathbf{2}$.

Similarly to what occurs with compound 7 , compounds 11, 12, 13 and 15 (Fig. 5) represent peaks with distinctly benzoxazole-like absorption spectra (Additional file 2: Figure S2) and masses coherent with the expected products $\left(\mathrm{m} / z 270.08,290.17,290.15\right.$ and $304.08[M+\mathrm{H}]^{+}$, respectively), but these could not be adequately purified nor spectroscopically characterized due to low yields or presence of concomitant impurities, therefore remaining as putative novel caboxamycin derivatives and candidates for future experiments.

\section{Biological activity}

All novel caboxamycin derivatives characterized in this work were tested against a selection of microorganisms (Gram-negative Escherichia coli, Gram-positive Staphylococcus aureus, Micrococcus luteus and S. albus, and yeast Candida albicans) via disk diffusion assay (Additional file 4: Table S12). Compounds $\mathbf{4}, \mathbf{8}$ and 9 were found to inhibit growth in all Gram-positive bacteria, with 9 showing an additional mild inhibition of $C$. albicans growth. On the other hand, compound $\mathbf{1 4}$ was shown to selectively inhibit the growth of $S$. aureus at $5 \mu \mathrm{g}$.

These compounds were likewise submitted to cytotoxicity assays against tumor cell lines A549 (lung), MDAMB-231 (breast), HT29 (colon), AGS (gastric) and A2780 (ovarian), using the murine fibroblast NIH/3T3 cell line as control (Additional file 4: Table S13). Most of the novel compounds show no activity under the selected cutoff level of $10 \mu \mathrm{M}$. However, compound 9 shows activity against all lines, which unfortunately includes the control cell line. On the other hand, compounds $\mathbf{4}$ and $\mathbf{8}$ could be of potential interest, as they seem to exert a selective cytotoxic effect against the gastric cell line AGS and ovarian cell line A2780 (compound 4), as well as a mild action against the lung cell line A549.

\section{Discussion}

The biosynthetic pathways of benzoxazole compounds nataxazole and caboxamycin show certain degree of flexibility since natural events of cross-talk with other pathways lead to hybrid compounds. In the case of nataxazole 
<smiles>COc1ccc(O)c(-c2nc3c(C(=O)O)cccc3o2)c1</smiles><smiles>Cc1ccc(O)c(-c2nc3c(C(=O)O)cccc3o2)c1</smiles><smiles>O=C(O)c1cccc2oc(-c3cc(Cl)ccc3O)nc12</smiles>

14<smiles>O=C(O)c1ccc(NC(=O)c2ncccc2O)cc1</smiles>

17<smiles>Cc1ccc(-c2nc3c(C(=O)O)cccc3o2)c(O)c1</smiles>

9<smiles>O=C(O)c1cccc2oc(-c3cccc(Cl)c3O)nc12</smiles>

12<smiles>COC(=O)c1cccc2oc(-c3c(O)cccc3Cl)nc12</smiles>

15<smiles>COC(=O)c1cccc2oc(-c3ncccc3O)nc12</smiles>

18<smiles>COC(=O)c1cccc2oc(-c3ccc(C)cc3O)nc12</smiles>

10<smiles>O=C(O)c1cccc2oc(-c3ccc(Cl)cc3O)nc12</smiles>

13<smiles>O=C(O)c1cccc2oc(-c3ncccc3O)nc12</smiles>

16<smiles>COC(=O)c1ccc(NC(=O)c2ncccc2O)cc1</smiles>

19

Fig. 5 Chemical structure of compounds 8-19 obtained by mutasynthesis in Streptomyces sp. NTK937 $\triangle \mathrm{cbxA} / \triangle \mathrm{entC}$

producer Streptomyces sp. Tü6176, the inactivation of polyketide synthase coding natPK led to the biosynthesis of benzoxazole UK-1 where the 6-methylsalicylic acid moiety had been substituted by salicylic acid, produced by the coelibactin biosynthesis pathway. Production of UK-1 can be improved growing the mutant strain in a zinc-deficient medium [8]. On the other hand, inactivation of $c b x A$, encoding a salicylate synthase, in caboxamycin producer Streptomyces sp. NTK937 led to the biosynthesis of $3^{\prime}$-hydroxycaboxamycin where the salicylate moiety had been substituted by 2,3-dihydroxybenzoate, produced by the enterobactin biosynthesis pathway [13]. The biosynthesis of these compounds represents a natural form of combinatorial production that prompted us to attempt the generation of novel benzoxazoles using combinatorial biosynthesis, heterologus expression and mutasynthesis, approaches that have been successful in many other cases [18-21].

We have generated eleven novel caboxamycin derivatives (compounds 4-6, 8-10, 12, 14 and 16-19), and five putative novel derivatives (compounds 7, 11-13 and 15), (Fig. 6). The usual yield for wild-type cultures under same conditions is $1-2.5 \mathrm{mg}$ for caboxamycin (1) and $0.2-0.7 \mathrm{mg}$ for $\mathrm{O}$-methylcaboxamycin (2). Compounds 4 and 5 (14.3 and $6.8 \mathrm{mg}$, respectively), generated by heterologous expression in S. albus J1074, were obtained in greater amounts due to the $e r m E^{*} \mathrm{p}$-enforced expression of the corresponding biosynthetic genes, and the lack of regulatory controls from its original strain. Compound 6, obtained by combinatorial biosynthesis in mutant strain $\Delta \mathrm{cbxA}(0.2 \mathrm{mg})$, was produced at levels coherent with those of wild-type strain Streptomyces sp. NTK 937 for O-methylcaboxamycin (2). In the case of compounds 8-10,14 and 16-19, these were isolated with varying success but at levels similar to those of Streptomyces sp. NTK 937, with higher quantities for the acid form than for the corresponding methyl ester. We also sought to improve mutasynthesis yields by ectopic expression of regulatory gene $c b x R$ in the strain $\triangle \mathrm{cbxA} / \Delta$ entC. However, production levels did not increase accordingly as in prior uses of $c b x R$ [13], possibly due to the lack of $c b x A$, which might contain the effecting region for $C b x R$, given the structural organization of the biosynthetic cluster. 


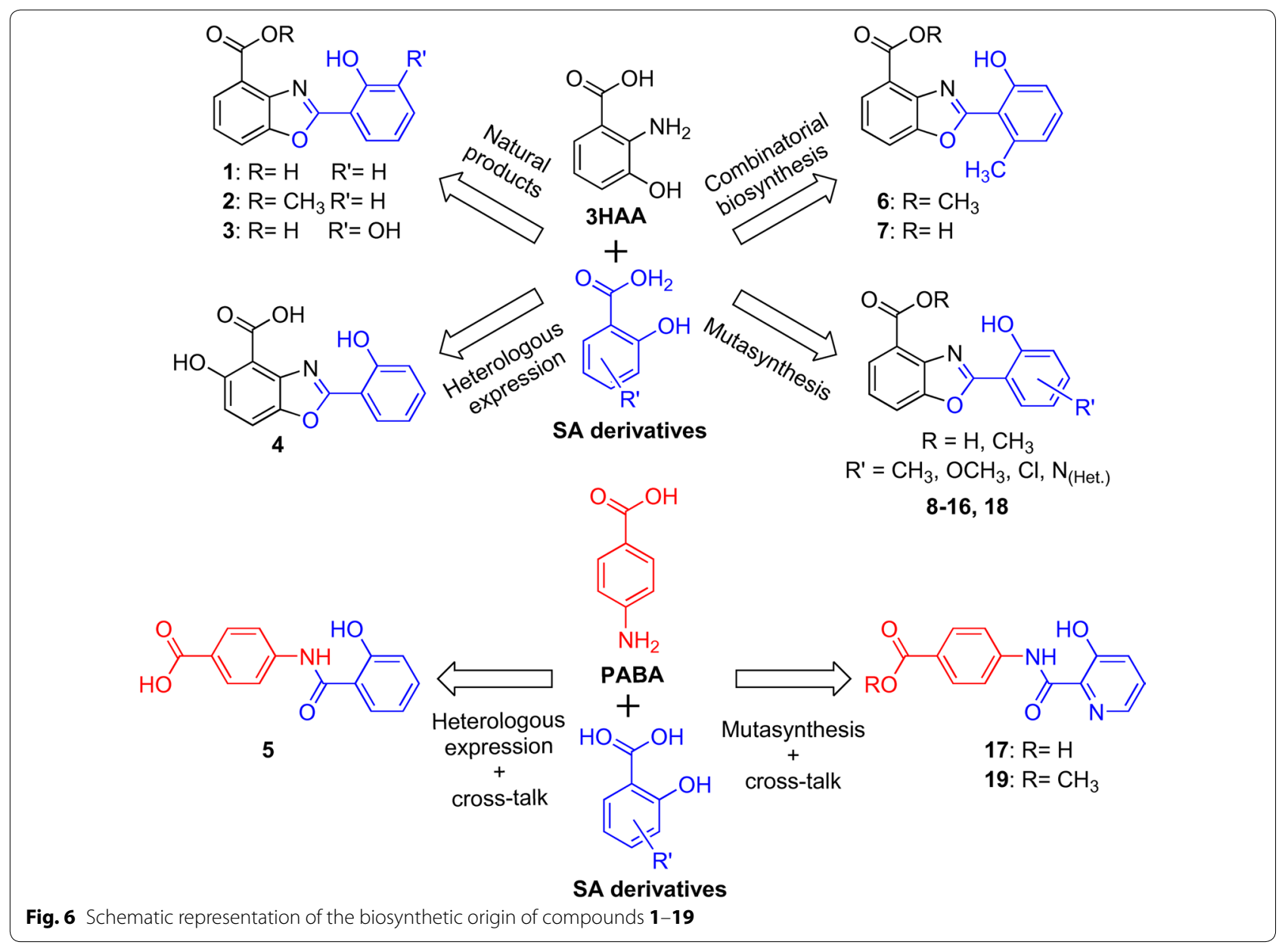

Some of these novel caboxamycin derivatives show improved antibiotic and cytotoxic activities (compounds 4, 8 and 9). Especially remarkable is the fact that 4 can actually inhibit the growth of $S$. albus, the host strain where the compound was produced by combinatorial biosynthesis, a feat similar to that observed in the heterologous expression of the nataxazole cluster, whose unmethylated product, AJI9561, showed deleterious effects on the growth of the host strain [7]. On the other hand, the production of compound $\mathbf{4}$ in $S$. albus clearly shows the existence of orthologs for $c b x F G H I$ within primary metabolism genes in $S$. albus strains, such as the biosynthetic pathway to aromatic amino acids [22, 23], secondary metabolism pathways, such as the biosynthesis of catecholate siderophores [24], or even alternative routes leading to $3 \mathrm{HAA}$ via $\mathrm{NAD}^{+}$biosynthesis pathway from tryptophan catabolism [25]. In addition, hydroxylation of the 3HAA moiety of $\mathbf{4}$ might be mediated by plm14 gene product, the immediate neighbor of the replaced genes in S. albus strain B29, which encodes a 3-hydroxybenzoate 6-hydroxylase activity that participates in paulomycins biosynthesis [16]. Otherwise, generation of $\mathbf{5}$ is serendipitous. It can only be surmised that stimulation of the chorismate pathway due to enforced salicylate biosynthesis by $c b x A$ overexpression may activate other chorismate-derived routes such as PABA biosynthesis (Fig. 6), which naturally takes part as the first step towards folate biosynthesis [26]. Furthermore, PABA is a direct precursor of candicidins, polyene polyketides produced by S. albus J1074 [15]. No gene can be held responsible for the amide bond formation, since, to the best of our knowledge, no similar PABA-derived compounds of biological origin have been described in the literature, and non-ribosomal amide links can be achieved in several different ways [27]. On the other hand, the amide bond of compound 5 (Fig. 6) could be generated by $\mathrm{CbxB}$ that exerts a similar role during caboxamycin biosynthesis [13].

The production of some caboxamycin derivatives has also allowed confirming the roles previously assigned to some nataxazole biosynthesis genes [7]. That is the case for the generation of compound $\mathbf{6}$ by heterologous 
expression of polyketide synthase coding natPK in Streptomyces sp. NTK937, and the subsequent improvement of its yield by expressing AMP-dependant synthetaseligase coding natL1, while the coexpression of natPK and nat 2 favored instead the production of 3 . These results are in consonance with the assigned roles of NatL1 and NatL2 in nataxazole biosynthesis: NatL2 was proposed to participate in the incorporation of the second 3HAA unit, while NatL1 might work on the condensation of 6MSA with 3HAA [7]. The small yields of 6 in $\Delta \mathrm{cbxA} /$ pnatPK and $\triangle \mathrm{cbxA} /$ pnatL2-PK might be due by the activity of $\mathrm{CbxC}$.

Finally, mutasynthesis experiments revealed some unexpected results. Curiously, the addition of 2,5-dihydroxybenzoate leads to the production of $\mathbf{1}$ and $\mathbf{2}$, through an unknown dehydroxylating mechanism that presumably acts after condensation of 2,5-dihydroxybenzoate to 3HAA, provided that no accumulation of SA occurs. Likewise, 3-methoxysalicylate gets spontaneously demethylated to DHB to become 3 , although in this instance, demethylation happens directly over the precursor, and DHB is indeed accumulated.

\section{Conclusions}

The diversity of benzoxazoles generated by mutasynthesis, together with those obtained by heterologous expression of caboxamycin biosynthesis genes $c b x A B$ $C D E$ in $S$. albus strains, and combinatorial biosynthesis experiments, show that the production of a wide variety of benzoxazoles could be potentially achieved by the sole expression of $c b x B C D E$ genes (or orthologs thereof), supplying an external source of salicylate-like compounds, or with the concomitant expression of other genes capable of synthesizing salicylates, such as $c b x A$ or natPK. The biological activity of the novel compounds generated, in comparison with that of the parental compound, can be altered and occasionally improved as shown in this work.

\section{Methods}

Strains, culture conditions and plasmids

Streptomyces sp. NTK937 [2], producer of caboxamycin, source of caboxamycin cluster genes $c b x A B C D E$; Streptomyces sp. NTK937 gene-deleted mutant strains $\triangle \mathrm{cbxA}$ and $\Delta \mathrm{cbxA} / \Delta \mathrm{entC}[13]$ were used respectively for heterologous expression of nataxazole cluster genes and for mutasynthesis; Streptomyces sp. Tü6176 [4], producer of nataxazole, source of natPK, natL1 and natL2 genes; $S$. albus J1074 [14] and S. albus B29 [15] were used as heterologous hosts for the expression of caboxamycin biosynthesis genes; E. coli DH10B (Invitrogen) and E. coli ET12567 (pUB307) [28] were used for subcloning and intergeneric conjugation, respectively.
Escherichia coli culture media LB and 2xTY were used as previously described [29]. Tryptone Soy Broth (TSB) was used for culture of Streptomyces spp., MA for conjugation and sporulation, and R5A for secondary metabolite production [30]. Mutasynthesis assays were carried out by adding a final concentration of $1 \mathrm{mM}$ of each salicylate-like compound (from a $1 \mathrm{M}$ stock, dissolved in DMSO) at the same time of inoculation with the bacterial pre-culture (Additional file 1: Figure S1).

Two plasmids were used: pSETeTc [7] for integrative expression of the $c b x A B C D E$ genes in $S$. albus strains, and pEM4T [31] for the expression of nataxazole cluster genes in $\triangle \mathrm{cbxA}$ strain. pCR-Blunt (Invitrogen) was used for routine PCR product cloning for verification purposes.

Culture media were supplemented with their due antibiotic when plasmid-bearing strains were used: ampicillin $(100 \mu \mathrm{g} / \mathrm{mL})$, apramycin $(100 \mu \mathrm{g} / \mathrm{mL}$ for E. coli, $25 \mu \mathrm{g} /$ $\mathrm{mL}$ for Streptomyces), thiostrepton $(50 \mu \mathrm{g} / \mathrm{mL})$, kanamycin $(25 \mu \mathrm{g} / \mathrm{mL})$, tetracycline $(10 \mu \mathrm{g} / \mathrm{mL})$, chloramphenicol $(25 \mu \mathrm{g} / \mathrm{mL})$, and/or nalidixic acid $(50 \mu \mathrm{g} / \mathrm{mL})$.

\section{DNA manipulation}

DNA manipulations were performed according to standard procedures for E. coli [29] and Streptomyces [28]. PCR amplifications were carried out with Herculase II Fusion DNA Polymerase (Agilent Technologies) following an optimized standard PCR procedure on a SureCycler 8800 thermocycler (Agilent Technologies): initial denaturation at $99.9^{\circ} \mathrm{C}$ for $2 \mathrm{~min}, 30$ cycles comprised of $99.9{ }^{\circ} \mathrm{C}$ denaturation for $10 \mathrm{~s}, 65^{\circ} \mathrm{C}$ annealing for $20 \mathrm{~s}$, and $72{ }^{\circ} \mathrm{C}$ elongation at $30 \mathrm{~s}$ per $\mathrm{kb}$ of DNA to be amplified, plus an extra final cycle of $72{ }^{\circ} \mathrm{C}$ for $3 \mathrm{~min}$. Products of the expected size were cloned into pCR-Blunt for sequence verification. PCR products were subsequently cloned into appropriate vectors using the selected restriction sites incorporated in the oligonucleotides.

\section{Construction of plasmids}

Plasmid pSET-cbxABCDE was built from a $6 \mathrm{~kb}$ PCR fragment containing caboxamycin genes $c b x A B C D E$. The PCR product was generated by using BamHIflanked SS/fw oligonucleotide (5'-AAGGATCCGATCT GCGACGTGTCGCCGTC-3') and EcoRI-flanked oligonucleotide AH/rv (5'-AGAATTCGGTGTTGTCGGG CATGGTG-3'). This fragment was subsequently purified, sequence-checked, and digested with the appropriate restriction enzymes, to be ligated into a BamHI/EcoRIdigested and dephosphorylated pSETeTc.

Constructions pnatPK and pnatL2-PK, both derived of pEM4T, for the expression of natPK or natL2, natX and natPK, respectively, were previously reported [9]. 
Plasmid pnatL1-PK, for simultaneous expression of natPK and natL1, was generated by blunt ended ligation of natL1 at the BamHI site of pnatPK. The natL1 gene was obtained as a PCR fragment using oligonucleotides ASL21/fw (5'-AGAATTCGTTCGTCTTCGGTCGGGA ATGCG-3') and ASL21/rv (5'-AAGGATCCGCGACCA TGTCCTGCTGATCGG-3').

\section{Analysis of metabolites by UPLC and HPLC-MS}

Cultures of selected strains or mutants were extracted with ethyl acetate containing $1 \%$ formic acid (to enhance the extraction of compounds containing ionizing groups) and analysed by reverse phase chromatography with an Acquity UPLC instrument fitted with a BEH C18 column $(1.7 \mu \mathrm{m}, 2.1 \times 100 \mathrm{~mm}$, Waters $)$, using acetonitrile $(\mathrm{AcN})$ and aqueous $0.1 \%$ trifluoroacetic acid (TFA) as eluents. The program uses an isocratic hold of $10 \% \mathrm{AcN}$ for $1 \mathrm{~min}$, followed by a linear gradient up to $100 \% \mathrm{AcN}$ over $7 \mathrm{~min}$, at a flow rate of $0.5 \mathrm{~mL} / \mathrm{min}$ and a column temperature of $35^{\circ} \mathrm{C}$.

For HPLC-MS analysis, an Alliance chromatographic system coupled to a ZQ4000 mass spectrometer and a SunFire C18 column $(3.5 \mu \mathrm{m}, 2.1 \times 150 \mathrm{~mm}$, Waters $)$ was used. Solvents were the same as above and elution was performed with an initial isocratic hold with $10 \% \mathrm{AcN}$ during 4 min followed by a linear gradient of AcN (10$88 \%$ ) over $30 \mathrm{~min}$, all at $0.25 \mathrm{~mL} / \mathrm{min}$. MS analysis was done by positive mode electrospray ionization (ESI), with a capillary voltage of $3 \mathrm{kV}$ and a cone voltage of $20 \mathrm{~V}$. Spectral identification and characterization of peaks was performed in both cases by photodiode array detection at $330 \mathrm{~nm}$, using Empower software (Waters) to extract bidimensional chromatograms at different wavelengths, depending on the spectral characteristics of the desired compound.

\section{Isolation and structural characterization of compounds}

Liquid production cultures were generally incubated at $30{ }^{\circ} \mathrm{C}$ and $250 \mathrm{rpm}$ for 7 days and then $1 \mathrm{~mL}$ samples from each of the flasks were extracted with an equal volume of acidified ethyl acetate. Solid production cultures were carried out using 25 -well plates with $1.5 \mathrm{~mL}$ solid R5A medium each and inoculated with a sterile cotton swab, then incubated at $30{ }^{\circ} \mathrm{C}$ for 7 days and extracted with an equal volume of acidified ethyl acetate. Both types of samples were subsequently vacuum-dried and redissolved in 50:50 DMSO:MeOH before chromatographic analysis.

Products 4-6, 8-10, 14 and 16-19 were isolated from $5 \times 400 \mathrm{~mL}$ (in $2 \mathrm{~L}$ flasks) cultures whose supernatants were first filtered, then concentrated on a C18 cartridge (10 g, Waters), and subsequently fractioned on a $0.1 \%$ TFA-MeOH gradient. Fractions were submitted to UPLC analysis, and those containing desired compounds were dried in vacuo, resuspended in 50:50 DMSO:MeOH, and processed on a preparative HPLC SunFire C18 column $(10 \mu \mathrm{m}, 10 \times 250 \mathrm{~mm}$, Waters) using experimentally determined isocratic mixtures of $0.05 \%$ TFA with either AcN or $\mathrm{MeOH}$ at $5 \mathrm{~mL} / \mathrm{min}$. The purity of the isolated peaks was determined by HPLC-MS before structural elucidation. The isolated compounds were then dried in vacuo, resuspended in 50:50 tert-butanol:water and lyophilised. Yield and productivity of each compound was as follows: 4, $14.3 \mathrm{mg}(7.15 \mu \mathrm{g} / \mathrm{mL}) ; \mathbf{5}, 6.8 \mathrm{mg}(4.2 \mu \mathrm{g} / \mathrm{mL}) ; \mathbf{6}, 0.2 \mathrm{mg}$ $(0.1 \mu \mathrm{g} / \mathrm{mL}) ; 8,1.6 \mathrm{mg}(0.8 \mu \mathrm{g} / \mathrm{mL}) ; \mathbf{9}, 2.1 \mathrm{mg}(1.05 \mu \mathrm{g} /$ $\mathrm{mL}) ; 10,0.5 \mathrm{mg}(0.25 \mu \mathrm{g} / \mathrm{mL}) ; 14,2.5 \mathrm{mg}(1.25 \mu \mathrm{g} / \mathrm{mL}) ; \mathbf{1 6}$, $1.3 \mathrm{mg}(0.65 \mu \mathrm{g} / \mathrm{mL}) ; 17,0.7 \mathrm{mg}(0.35 \mu \mathrm{g} / \mathrm{mL}) ; 18,0.3 \mathrm{mg}$ $(0.15 \mu \mathrm{g} / \mathrm{mL})$; and 19, $0.5 \mathrm{mg}(0.15 \mu \mathrm{g} / \mathrm{mL})$.

Structural elucidation was carried out at Fundación Medina (Granada, Spain) by a combination of ${ }^{1} \mathrm{H},{ }^{13} \mathrm{C}$, COSY and HSQC experiments using DMSO- $d_{6}$ as solvent (Additional file 2: Figure S2, Tables S1-S11). LCDAD-HRMS analysis was carried out on an Agilent 1200 Rapid Resolution HPLC system coupled to a Bruker maXis mass spectrometer. For the NMR analysis the samples were dissolved in DMSO- $d_{6}$ and transferred to a $1.7 \mathrm{~mm}$ tube. Acquisitions were carried out on a Bruker AVANCE III $500 \mathrm{MHz}$ spectrometer equipped with a $1.7 \mathrm{~mm}$ TCI Microcryoprobe. All spectra $\left({ }^{1} \mathrm{H},{ }^{13} \mathrm{C}, \mathrm{COSY}\right.$, HSQC, HMBC) were registered at $24{ }^{\circ} \mathrm{C}$ (Additional file 3: Figures S3-S13).

\section{Bioactivity assays}

The antibiotic activities of $4-6,8-10,14$ and $16-19$ were analyzed with an antibiotic disk diffusion assay against $S$. albus J1074, E. coli, S. aureus and M. luteus. The antifungal activity was tested against $C$. albicans. In all cases 1 , $2.5,5,10$, and $20 \mu \mathrm{g}$ of each compound were used and loaded on $6 \mathrm{~mm}$ paper disks. Plates were incubated overnight at $37^{\circ} \mathrm{C}$ for E. coli, S. aureus and M.luteus, and at $30{ }^{\circ} \mathrm{C}$ for S. albus J1074 and C. albicans (Additional file 4: Table S12).

Cytotoxic activity of compounds 4-14 was tested against the following human tumor cell lines: colon adenocarcinoma (HT29), non-small cell lung cancer (A549), breast adenocarcinoma (MDA-MB-231), gastric carcinoma (AGS), and ovarian carcinoma (A2780). Mouse embryonic fibroblast cell line NIH/3T3 was used as control to evaluate cytotoxicity against non-malignant cells (Additional file 4: Table S13). Cells were previously grown for a week on DMEM-10\%FBS medium, then aliquoted to 5000 cells per well in 96-well plates using the Cell counting kit-8-(96992) (Sigma-Aldrich) and grown for an extra $24 \mathrm{~h}$. Compounds were dissolved in DMSO, keeping in mind that final concentration of DMSO in the assays should be kept at $0.1 \%$. After the incubation, $10 \mu \mathrm{L}$ 
of compound (in diverse concentrations) were added to each well and incubated for another $48 \mathrm{~h}$. Lastly, $10 \mu \mathrm{L}$ of CCK-8 reagent (Sigma-Aldrich) were added, left to develop for $2 \mathrm{~h}$ in the incubator, and measured at $450 \mathrm{~nm}$ using an Elisa Bio-tek ELx 800 (BioTek).

\section{Additional files}

Additional file 1. Compounds used for mutasynthesis experiments.

Additional file 2. Spectroscopic data of compounds characterized in this work.

Additional file 3. NMR spectra of compounds characterized in this work.

Additional file 4. Biological assays results.

\section{Authors' contributions}

JAS, CM and CO conceived and designed the project; AAL conducted experiments; $A A L$ and $C O$ analyzed the data and drafted the manuscript. AAL, CM, $\mathrm{JAS}$, and CO contributed to preparing the final version of the paper. All authors read and approved the final manuscript.

\section{Acknowledgements}

We thank Dr. Fernando Reyes from Fundación Medina for technical support in the structural elucidation of compounds, and to Hans-Peter Fiedler (University of Tübingen, Germany) for providing us the caboxamycin producer. We thank Fundación Bancaria Cajastur for financial support to C.O.

\section{Competing interests}

The authors declare that they have no competing interests.

\section{Availability of data and materials}

The datasets on which the findings and conclusions of this article is based upon are all included in this manuscript and the Additional files 1, 2, 3, 4 associated with it.

\section{Funding}

This research was supported by a grant of the Spanish Ministry of Economy and Competitiveness (MINECO) (BIO2012-33596 to J.A.S.). A.A.L. was the recipient of a predoctoral fellowship of FICYT (Asturias).

\section{Publisher's Note}

Springer Nature remains neutral with regard to jurisdictional claims in published maps and institutional affiliations.

Received: 20 April 2017 Accepted: 23 May 2017

Published online: 25 May 2017

\section{References}

1. Olano C, Cano-Prieto C, Losada AA, Bull AT, Goodfellow M, Fiedler HP, Méndez C, Salas JA. Draft genome sequence of marine actinomycete Streptomyces sp. strain NTK937, producer of the benzoxazole antibiotic caboxamycin. Genome Announc. 2014;2:2-3.

2. Hohmann C, Schneider K, Bruntner C, Irran E, Nicholson G, Bull AT, Jones AL, Brown R, Stach JEM, Goodfellow M, Beil W, Krämer M, Imhoff JF, Süssmuth SD, Fiedler HP. Caboxamycin, a new antibiotic of the benzoxazole family produced by the deep-sea strain Streptomyces sp. NTK 937. J Antibiot. 2009:62:99-104.

3. Reed PW, Lardy HA. A23187: a divalent cation ionophore. J Biol Chem. 1972;247:6970-7.

4. Sommer PS, Almeida RC, Schneider K, Beil W, Süssmuth RD, Fiedler HP. Nataxazole, a new benzoxazole derivative with antitumor activity produced by Streptomyces sp. Tü 6176. J Antibiot. 2008;61:683-6.
5. Michel KH, Boeck LD, Hoehn MM, Jones ND, Chaney MO. The discovery, fermentation, isolation, and structure of antibiotic A33853 and its tetraacetyl derivative. J Antibiot. 1984;37:441-5.

6. Wu Q, Liang J, Lin S, Zhou X, Bai L, Deng Z, Wang Z. Characterization of the biosynthesis gene cluster for the pyrrole polyether antibiotic calcimycin (A23187) in Streptomyces chartreusis NRRL 3882. Antimicrob Agents Chemother. 2011:55:974-82.

7. Cano-Prieto C, García-Salcedo R, Sánchez-Hidalgo M, Braña AF, Fiedler HP, Méndez C, Salas JA, Olano C. Genome mining of Streptomyces sp. Tü6176: characterization of the nataxazole biosynthesis pathway. ChemBioChem. 2015:16:1461-73.

8. Cano-Prieto C, Losada AA, Braña AF, Méndez C, Salas JA, Olano C. Crosstalk of nataxazole pathway with chorismate-derived ionophore biosynthesis pathways in Streptomyces sp. Tü 6176. ChemBioChem. 2015;16:1925-32

9. Lv M, Zhao J, Deng Z, Yu Y. Characterization of the biosynthetic gene cluster for benzoxazole antibiotics A33853 reveals unusual assembly logic. Chem Biol. 2015;22:1313-24.

10. Demmer CS, Bunch L. Benzoxazoles and oxazolopyridines in medicinal chemistry studies. Eur J Med Chem. 2015;97:778-85.

11. Tipparaju SK, Joyasawal S, Pieroni M, Kaiser M, Brun R, Kozikowski AP. In pursuit of natural product leads: synthesis and biological evaluation of 2-[3-hydroxy-2-[(3-hydroxypiridine-2-carbonyl)amino]phenyl] benzoxazole-4-carboxylic acid (A-33853) and its analogues: discovery of $\mathrm{N}$-(2-benzoxazol-2-ylphenyl)benzamides as novel antileishmanial chemotypes. J Med Chem. 2008;51:7344-7

12. Ward DN, Talley DC, Tavag M, Menji S, Schaughency P, Baier A, Smith PJ. UK-1 and structural analogs are potent inhibitors of hepatitis $C$ virus replication. Bioorg Med Chem Lett. 2014;24:609-12.

13. Losada AA, Cano-Prieto C, García-Salcedo R, Braña AF, Méndez C, Salas JA Olano C. Caboxamycin biosynthesis pathway and identification of novel benzoxazoles produced by cross-talk in Streptomyces sp. NTK937. Microb Biotechnol. 2017. doi:10.1111/1751-7915.12716.

14. Chater KF, Wilde LC. Restriction of a bacteriophage of Streptomyces albus G involving endonuclease sall. J Bacteriol. 1976;128:644-50.

15. Olano C, García I, González A, Rodriguez M, Rozas D, Rubio J, SánchezHidalgo M, Braña AF, Méndez C, Salas JA. Activation and identification of five clusters for secondary metabolites in Streptomyces albus J1074. Microb Biotechnol. 2014;7:242-56.

16. González A, Rodríguez M, Braña AF, Méndez C, Salas JA, Olano C. New insights into paulomycin biosynthesis pathway in Streptomyces albus $J 1074$ and generation of novel derivatives by combinatorial biosynthesis. Microb Cell Fact. 2016;15:56

17. Bibb MJ, Janssen GR, Ward JM. Cloning and analysis of the promoter region of the erythromycin resistance gene (ermE) of Streptomyces erythraeus. Gene. 1985;38:215-26.

18. Olano C, Méndez C, Salas JA. Molecular insights on the biosynthesis of antitumour compounds by actinomycetes. Microb Biotechnol. 2011:4:144-64.

19. Wilkinson B, Gregory MA, Moss SJ, Carletti I, Sheridan RM, Kaja A, Ward M, Olano C, Mendez C, Salas JA, Leadlay PF, vanGinckel R, Zhang MQ. Separation of anti-angiogenic and cytotoxic activities of borrelidin by modification at the C17 side chain. Bioorg Med Chem Lett. 2006;16:5814-7.

20. Sialer C, García I, González-Sabín J, Braña AF, Méndez C, Morís F, Salas JA. Generation by mutasynthesis of potential neuroprotectant derivatives of the bipyridyl collismycin A. Bioorg Med Chem Lett. 2013;23:5707-9.

21. Gou L, Wu Q, Lin S, Li X, Liang J, Zhou X, An D, Deng Z, Wang Z. Mutasynthesis of pyrrole spiroketal compound using calcimycin 3-hydroxy anthranilic acid biosynthetic mutant. Appl Microbiol Biotechnol. 2013.97:8183-91.

22. Morollo AA, Bauerle R. Characterization of composite aminodeoxyisochorismate synthase and aminodeoxyisochorismate lyase activities of anthranilate synthase. Proc Natl Acad Sci USA. 1993;90:9983-7.

23. Light $\mathrm{SH}$, Anderson WF. The diversity of allosteric controls at the gateway to aromatic amino acid biosynthesis. Protein Sci. 2013;22:395-404.

24. Walsh CT, Liu J, Rusnak F, Sakaitani M. Molecular studies on enzymes in chorismate metabolism and the enterobactin biosynthetic pathway. Chem Rev. 1990;90:1105-29.

25. Gazzaniga F, Stebbins R, Chang SZ, McPeek MA, Brenner C. Microbial NAD metabolism: lessons from comparative genomics. Microbiol Mol Biol Rev. 2009:73.529-41. 
26. de Crécy-Lagard V, El Yacoubi B, de la Garza RD, Noiriel A, Hanson AD. Comparative genomics of bacterial and plant folate synthesis and salvage: predictions and validations. BMC Genom. 2007;8:245.

27. Goswami A, van Lanen SG. Enzymatic strategies and biocatalysts for amide bond formation: tricks of the trade outside of the ribosome. Mol Biosyst. 2015;11:338-53.

28. Kieser T, Bibb MJ, Buttner MJ, Chater KF, Hopwood DA. Practical Streptomyces genetics. Norwich: The John Innes Foundation; 2000.

29. Green MR, Sambrook J. Molecular cloning: a laboratory manual. New York Cold Spring Harbor Press; 2012.
30. Fernández E, Weissbach U, Sánchez Reillo C, Braña AF, Méndez C, Rohr J, Salas JA. Identification of two genes from Streptomyces argillaceus encoding glycosyltransferases involved in transfer of a disaccharide during biosynthesis of the antitumor drug mithramycin. J Bacteriol. 1998;180:4929-37.

31. Menéndez N, Nur-e-Alam M, Fischer C, Braña AF, Salas JA, Rohr J, Ménde C. Deoxysugar transfer during chromomycin A3 biosynthesis in Streptomyces griseus subsp. griseus: new derivatives with antitumor activity. Appl Environ Microbiol. 2006;72:167-77.

\section{Submit your next manuscript to BioMed Central and we will help you at every step:}

- We accept pre-submission inquiries

- Our selector tool helps you to find the most relevant journal

- We provide round the clock customer support

- Convenient online submission

- Thorough peer review

- Inclusion in PubMed and all major indexing services

- Maximum visibility for your research

Submit your manuscript at

www.biomedcentral com/submit 